\title{
Holographic characteristics of a 1-mm-thick photopolymer to be used in holographic memories
}

\author{
Manuel Ortuño, Sergi Gallego, Celia García, Cristian Neipp, and Inmaculada Pascual
}

\begin{abstract}
Poly(vinyl alcohol-acrylamide) photopolymers are materials of interest in the field of digital information storage (holographic memories). We analyzed the behavior of a 1-mm-thick photopolymer. Using a standard holographic setup, we recorded unslanted diffraction gratings. The material has high angular selectivity $\left(0.4^{\circ}\right)$, good sensitivity $\left(88 \mathrm{~mJ} / \mathrm{cm}^{2}\right)$, and small losses caused by absorption and scattering of light. It also has a high maximum diffraction efficiency (70\%). A significant induction period was seen in the material. The authors hypothesize that, during most of this induction period, polymerization does in fact take place but is not reflected in the appearance of the diffracted light until a certain threshold value of exposure is reached. (C) 2003 Optical Society of America
\end{abstract}

OCIS codes: $\quad 090.2900,090.4220,090.7330$.

\section{Introduction}

Photopolymers ${ }^{1}$ are one of the many different types of recording material used in holography. These materials are undergoing intense study because important practical applications may result from their development, especially in the field of digital information storage, in holographic memories. ${ }^{2}$ This type of device makes use of the thickness of the recording material to record data, a characteristic that is inherent in holography, as opposed to currently available optical devices such as CDs and DVDs in which use is made of the surface only. For this reason the theoretical information storage capacity of holographic memories is much greater than that of current techniques; therefore, it is necessary to develop thick photopolymers with good holographic properties. ${ }^{3}$

Photopolymers based on poly(vinyl alcoholacrylamide) are one type of material of interest ${ }^{4}$ ow- $^{-}$ ing to their good properties, in particular, their mean energetic sensitivity compared with that of other available materials; the possibility of easily adapting

M. Ortuño, S. Gallego, C. García, and I. Pascual (pascual@ua.es) are with the Departamento Interuniversitario de Óptica, Universidad de Alicante, Apartado 99, E-03080 Alicante, Spain. C. Neipp is with the Departamento de Física, Ingeniería de Sistemas y Teoría de la Señal, Universidad de Alicante, Apartado 99, E-03080 Alicante, Spain.

Received 25 March 2003; revised manuscript received 28 July 2003.

0003-6935/03/357008-05\$15.00/0

(C) 2003 Optical Society of America their spectral sensitivity to the type of recording laser used by simple changing of the sensitizer dye; and their high diffraction efficiency, together with acceptable resolution and signal-to-noise ratio. Their low cost and ease of preparation are also important, and these characteristics may even be decisive when one is choosing the recording material to be used in a practical application..$^{4,5}$

In this study we analyze the behavior of an approximately 1-mm-thick poly(vinyl alcohol-acrylamide) photopolymer with a view to its possible use in writeonce read-many holographic memories. Using a standard holographic setup, we recorded unslanted diffraction gratings in the material and determined the response of the photopolymer in terms of several holographic parameters: diffraction efficiency, sensitivity, and angular response. In particular, we analyzed the peculiarities of this type of photopolymer when thick layers were used.

\section{Experiment}

\section{A. Preparation of the Recording Material}

Using the force of gravity, we deposited the solution whose composition is listed in Table 1 in polystyrene molds and left them in the dark. When part of the water had evaporated, the dry material was removed from the mold, cut into squares, and attached, without the need for adhesive, to the surfaces of glass plates that measured $6.5 \mathrm{~cm} \times 6.5 \mathrm{~cm}$. The plates were then ready for exposure, which took place immediately. The thickness of the material was measured with a conventional micrometer. ${ }^{6}$ 
Table 1. Composition of the Solution Used to Prepare Layers of the Recording Material Relative to the Thickness To Be Obtained

\begin{tabular}{lcc}
\hline \multicolumn{1}{c}{ Component } & \multicolumn{2}{c}{ Composition } \\
\cline { 2 - 3 } & $800-1000-\mu$ m Layer & 7.00 \\
\hline Poly(vinyl alcohol) Fluka 18-88 (\% weight per volume) & 13.30 & 0.20 \\
Triethanolamine (M) & 0.15 & 0.44 \\
Acrylamide (M) & 0.34 & $2.44 \times 10^{-4}$ \\
Yellowish eosin (M) & $9.00 \times 10^{-5}$ & 2000 \\
\hline
\end{tabular}

\section{B. Holographic Setup}

The experimental device is shown in Fig. 1. An argon laser at a wavelength of $514 \mathrm{~nm}$ was used to store unslanted diffraction gratings by means of continuous laser exposure. The laser beam was split into two secondary beams with an intensity ratio of $1: 1$. The diameters of these beams were increased to 1.5 cm with an expander, while spatial filtering was ensured. The object and the reference beams were recombined at the sample at an angle of $16.8^{\circ}$ to the normal by an appropriate set of mirrors, and the spatial frequency obtained was 1125 lines $/ \mathrm{mm}$. The working intensity at $514 \mathrm{~nm}$ was $5 \mathrm{~mW} / \mathrm{cm}^{2}$. The diffracted and transmitted intensities were monitored in real time with a $\mathrm{He}-\mathrm{Ne}$ laser positioned at Bragg's angle $\left(20.8^{\circ}\right)$ tuned to $633 \mathrm{~nm}$, where the material is not sensitive. To obtain transmission and diffraction efficiency as a function of the angle at reconstruction we placed the plates upon a rotating stage. The transmission and diffraction efficiencies (TE and DE, respectively) were calculated as the ratio of the transmitted and the diffracted beam, respectively, to the incident power.

\section{Results}

Figure 2 graphs the diffraction efficiency and the DE plus TE relative to exposure $E$ for a $1000-\mu \mathrm{m}$-thick layer of photopolymer. The sum of DE and TE provides an idea of the amount of light that is lost to absorption and scattering by the glass plate and the recording material. This value was seen to be 78 $84 \%$ during recording of the diffraction grating. Therefore, we are dealing with a high value that furthermore remains relatively constant.

The maximum DE obtained during recording, $\sim 70 \%$, is also high, and this large value is necessary

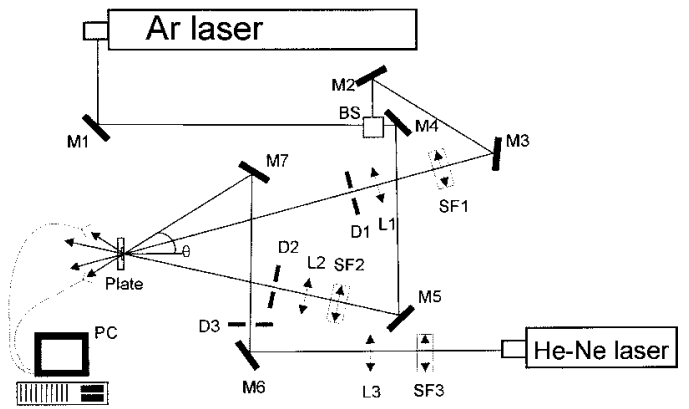

Fig. 1. Experimental setup: $\quad \mathrm{BS}$, beam splitter, $\mathrm{M}_{i}$, mirrors; $\mathrm{SF}_{i}$, spatial filters; $\mathrm{L}_{i}$, lenses; $\mathrm{D}_{i}$, diaphragms; $\mathrm{PC}$, data recorder. both in the recording of multiple holograms with low individual DE and in angular multiplexing, ${ }^{7}$ a technique that can be used in practical developments of holographic memories. The right most points in Fig. 2 correspond to the end of the recording stage.

We then obtained the angular response shown in Fig. 3 by reconstructing the diffraction grating. It can be seen that the maximum diffraction efficiency corresponds to a very narrow margin of only $0.4^{\circ}$, which implies high angular selectivity; this is an important quality for holographic memory applications and is not found in thin materials. ${ }^{8}$

In addition, it can also be seen (Fig. 2) that a minimum energy is necessary for the material to respond (when $E<20 \mathrm{~mJ} / \mathrm{cm}^{2}$ there is no diffracted beam), and this behavior becomes more pronounced when the monomer concentration in the composition is less than optimum (Fig. 4). This induction period is not seen when a recording laser beam of the same incident intensity is used with standard layers $100 \mu \mathrm{m}$ or less thick, ${ }^{8,9}$ as can be seen from Fig. 5. However, such an induction period is seen when the incident intensity is low. ${ }^{10}$ The induction period is often related to the presence of inhibitors. ${ }^{11}$ Trace quantities of transition elements that are present as impurities in the monomer used in the composition of the photopolymer or the presence of oxygen that is occluded in the material may deactivate the excited state of the dye or the polymer chains that are being formed. ${ }^{12}$ Specifically, oxygen is known to be an inhibitor of radical polymerization because it bonds with the propagating radicals to form peroxide radi-

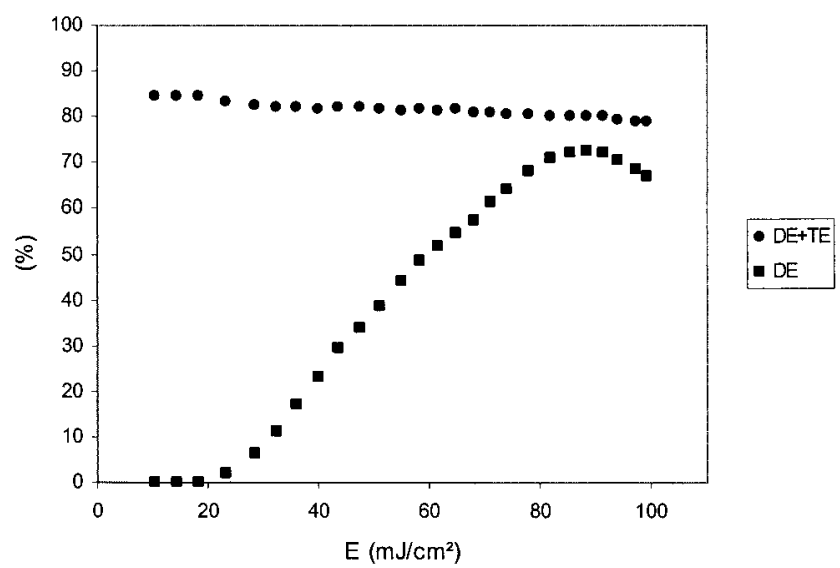

Fig. 2. DE and DE plus TE versus exposure for a 1000- $\mu \mathrm{m}$-thick photopolymer layer. 


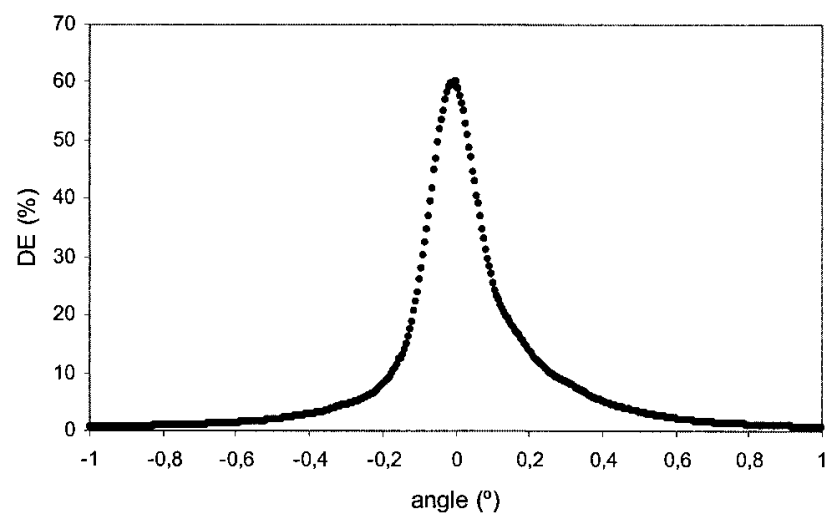

Fig. 3. DE versus angle for a $1000-\mu \mathrm{m}$-thick photopolymer layer.

cals of low reactivity. ${ }^{11}$ Once the inhibitor has been used up, polymerization progresses normally. This effect is important in liquid-phase polymerization in stirred systems in which the whole volume reacts simultaneously. This induction period is also seen in liquid photopolymers. ${ }^{13}$ The existence of an induction period in the material studied suggests that an inhibitor is present. However, the fact that this period is longer if the monomer concentration is low and is not seen in thin layers unless the incident intensity of the recording laser is reduced by $90 \%$ casts doubt on the validity of this hypothesis. The total quantity of oxygen and trace elements depends on the thickness of the material, but such is not true of their concentration. In a thick material the concentration of impurities is lower because of the lower concentration of monomer in the optimized composition (Table 1), and it is even lower for the concentration shown in Fig. 4, whereas the oxygen concentration is of the same order of magnitude. However, the induction period is longer in thick layers and increases when the monomer concentration is decreased. In solid-layer photopolymers diffusion of the species is much more restricted than in liquid systems, and the material polymerizes from the surface inward. This means that the effect of the con-

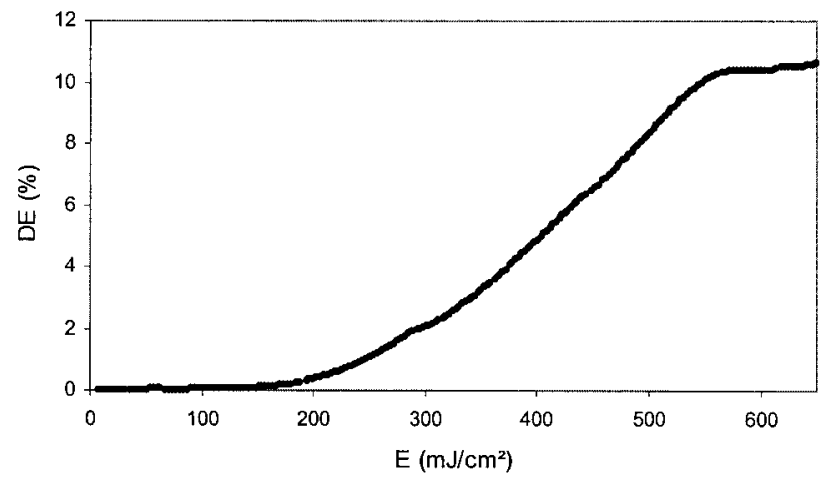

Fig. 4. DE versus exposure $E$ for a $1000-\mu \mathrm{m}$-thick photopolymer layer with a low acrylamide concentration $(0.17 \mathrm{M}$ in the original solution).

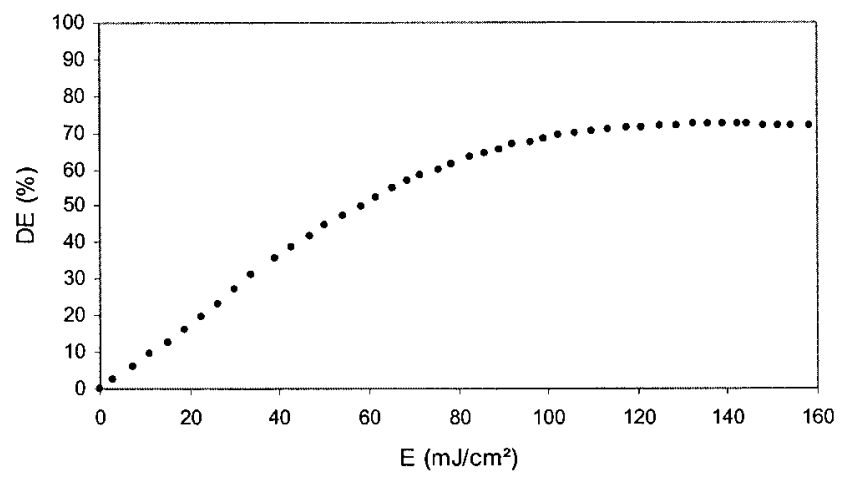

Fig. 5. DE versus exposure $E$ for a $100-\mu m$-thick photopolymer layer.

centration of the components in the material should be taken into account rather than their total amount.

If we consider the type of polymerization that is taking place and the concentration of possible inhibitors in relation to the thickness of the material and the monomer concentration, together with the facts that there is an induction period in thin layers only when the incident intensity is low and that the sensitivity of thick layers, in spite of the induction period, does not change compared with that of thinner layers (Table 2), it is possible to provide an alternative approach to explain the induction period. In particular, we believe not that it is a true induction period, during which polymerization is inhibited, but rather that during this time polymer chains are being formed that, however, do not give rise to an increase in diffraction efficiency. This could be so because, for a thick material, optimization of its composition requires a dye concentration almost 10 times less than that used in thin plates (Table 1). This means that the mean free distance covered by the photons of light before they collide with a dye molecule is greater in thick layers. Therefore the oligomers formed initially may be far apart and may not form a consistent grating; therefore there is no diffracted beam until a threshold value of exposure is reached. Inhibition reactions would therefore account for only a small fraction of the total induction period observed. This hypothesis is in agreement with the fact that there is an induction period in thin layers when the incident intensity is low; because the rate of polymerization is reduced, the grating is formed more slowly, and so more energy is necessary to produce an appreciable diffraction efficiency. The increase in the induction period in thick layers when the monomer concentra-

Table 2. Exposure $E$ Necessary for $60 \%$ and $70 \%$ Diffraction Efficiency Relative to Thickness of the Photopolymer Layer

\begin{tabular}{ccc}
\hline & \multicolumn{2}{c}{$E\left(\mathrm{~mJ} / \mathrm{cm}^{2}\right)$} \\
\cline { 2 - 3 } Thickness of Layer $(\mu \mathrm{m})$ & $\mathrm{DE}=60 \%$ & $\mathrm{DE}=70 \%$ \\
\hline 100 & 76 & 110 \\
1000 & 71 & 78 \\
\hline
\end{tabular}




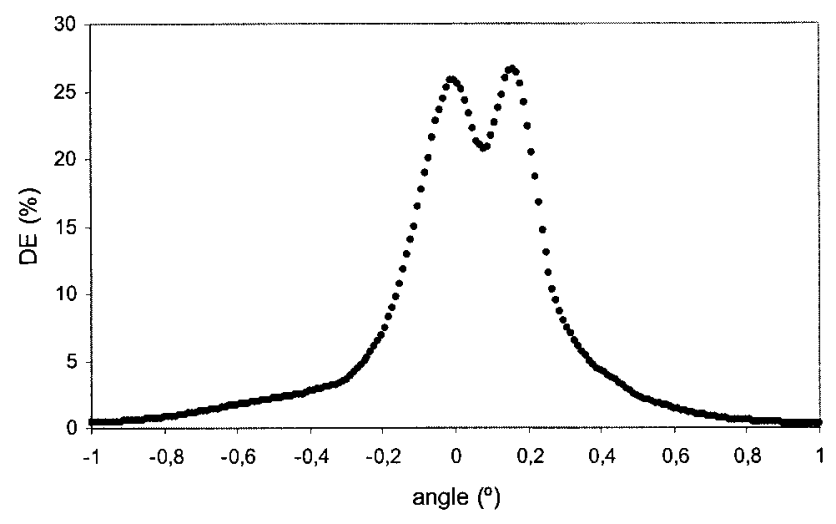

Fig. 6. DE versus angle for an overexposed photopolymer layer.

tion is decreased may also be explained by a slower rate of polymerization, with the result that the grating formed is not sufficiently consistent until $E$ reaches a certain threshold value, enabling the diffraction efficiency to increase significantly. The fact that the sensitivity of the material is not substantially changed for thick layers, in spite of there being a long induction period, also supports the idea that polymerization does in fact take place during this period.

Another effect to be considered can be seen from Fig. 6, which shows the angular response of a diffraction grating stored in an 800- $\mu \mathrm{m}$-thick layer. Here the phenomenon of splitting of the maximum DE can be seen, and this may be attributed to the presence of simultaneously recorded scatter noise gratings, as described in relation to other recording materials. ${ }^{14}$ The shape of the angular response curve and Fig. 7, which shows the sum of $\mathrm{DE}$ and TE versus exposure $E$, support this hypothesis because the sum is $76 \%-$ $55 \%$, which corresponds to almost double the loss that occurs in layers in which this phenomenon is not observed (Fig. 2). This behavior is clearly seen when the material is overexposed, as in this case, in which $E=1000 \mathrm{~mJ} / \mathrm{cm}^{2}$ at the end of the recording stage,

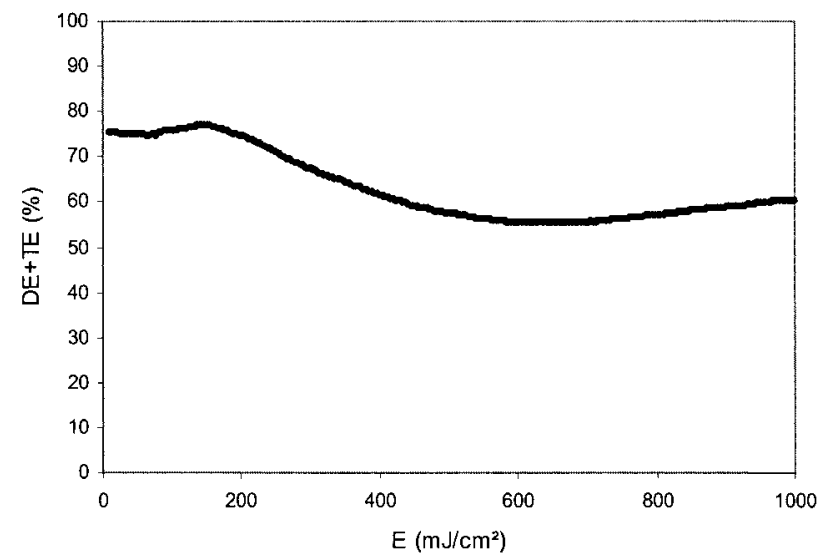

Fig. 7. DE plus TE versus exposure $E$ for an over-exposed photopolymer layer.

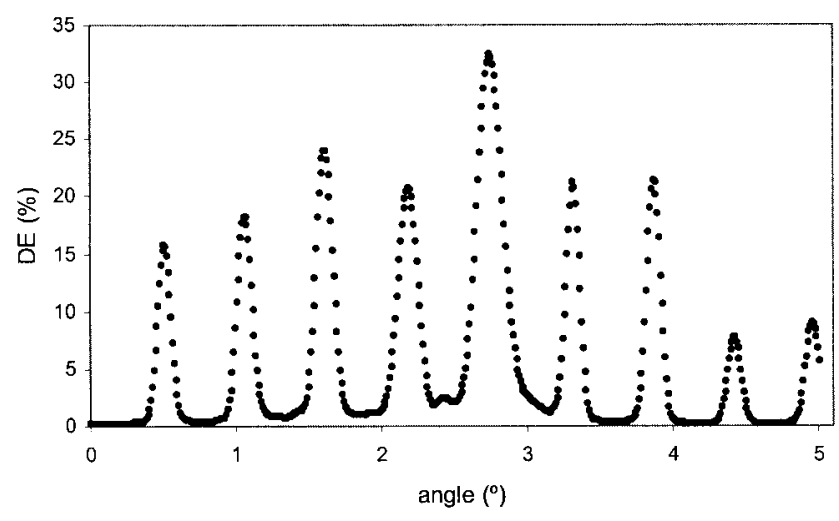

Fig. 8. DE versus angle during the recording of various diffraction gratings by angular multiplexing.

which represents a 10 -fold increase relative to the optimum exposure $\left(E=100 \mathrm{~mJ} / \mathrm{cm}^{2}\right)$.

Finally, to evaluate the behavior of thick photopolymer layers in terms of their possible application relative to holographic memories, in Fig. 8 we have recorded nine diffraction gratings in the same zone of a 1000- $\mu$ m-thick recording material, using the angular multiplexing technique and reducing by $0.5^{\circ}$ the angle between the perpendicular to the photopolymer layer and the initial position at the Bragg angle, between recording one diffraction grating and the next. In this way a variation of $\pm 2.5^{\circ}$ in rotation of the axis perpendicular to the work bench is sufficient to include the nine gratings. We obtained a high value for each individual maximum $\mathrm{DE}$ and sufficient separation between the gratings to permit their independent reconstruction. The number $M$ obtained in these conditions was $M \#=38$, which represents a good dynamic range. ${ }^{15}$

\section{Conclusions}

We have analyzed the holographic behavior of a thick poly(vinyl alcohol-acrylamide) photopolymer with a view to its application in holographic memories. The material has high angular selectivity $\left(0.4^{\circ}\right)$, good sensitivity $\left(88 \mathrm{~mJ} / \mathrm{cm}^{2}\right)$, and small losses to absorption and scattering of light. It also has a high maximum diffraction efficiency (70\%, reaching $80 \%$ if a correction is made for Fresnel losses). There was seen to be a significant induction period in the material. We analyzed this phenomenon in relation to the presence of inhibitors in the composition of the material. Comparison with thin materials seems to support the idea of a false induction period, which may be a different phenomenon from that which occurs in classic polymerization caused by the presence of inhibitors. The authors have put forward the hypothesis that, during most of this induction period, polymerization does in fact take place but is not reflected in the appearance of diffracted light until a certain threshold value of exposure is reached. This phenomenon may be explained by the low dye concentration in this type of composition, which, during the initial moments of exposure, gives rise to an in- 
consistent polymer grating that does not produce a change in refractive index in the exposed zones; therefore no diffraction of light is produced.

In addition, we observed the appearance of noise gratings. This phenomenon occurs when the recording material is overexposed and gives rise to a typical split of the maximum DE on the angular response curve, an effect that does not occur with the correct exposure.

We used a simple angular multiplexing technique to evaluate the behavior of the material during the recording of multiple diffraction gratings and obtained an angular response with high individual values of $\mathrm{DE}$, adequate separation between the stored diffraction gratings, and a good dynamic range $(M \#=$ $38)$.

This research was supported by the Ministerio de Ciencia y Tecnología, Comisión Interministerial de Ciencia y Tecnología, Spain, under project MAT20001361-C04-04 and by Generalitat Valenciana, Spain, project GV01-130.

\section{References}

1. R. A. Lessard and G. Manivannan, "Holographic recording materials: an overview," in Holographic Materials, T. J. Trout, ed., Proc. SPIE 2405, 2-23 (1995).

2. R. M. Shelby, J. A. Hoffnagle, G. W. Burr, C. M. Jefferson, M.-P. Bernal, H. Coufal, R. K. Grygier, H. Günther, R. M. Macfarlane, and G. T. Sincerbox, "Pixel-matched holographic data storage with megabit pages," Opt. Lett. 22, 1509-1511 (1997).

3. H.-Y. S. Li and D. Psaltis, "Three-dimensional holographic disks," Appl. Opt. 33, 3764-3774 (1994).
4. J. R. Lawrence, F. T. O’Neill, and J. T. Sheridan, "Photopolymer holographic recording material," Optik (Stuttgart) 112, 449-463 (2001).

5. S. Martin, C. A. Feely, and V. Toal, "Holographic recording characteristics of an acrylamide-based photopolymer," Appl. Opt. 36, 5757-5769 (1997).

6. M. Ortuño, S. Gallego, C. Neipp, A. Beléndez, and I. Pascual, "Optimization of a $1 \mathrm{~mm}$ thick PVA/acrylamide recording material to obtain holographic memories: method of preparation and holographic properties," Appl. Phys. B 76, 851-857 (2003).

7. J. H. Hong, I. McMichael, T. Y. Chang, W. Christian, and E. G. Paek, "Volume holographic memory systems: techniques and architectures," Opt. Eng. 34, 2193-2203 (1995).

8. S. Gallego, M. Ortuño, C. Neipp, C. García, A. Beléndez, and I. Pascual, "Overmodulation effects in volume holograms recorded on photopolymers," Opt. Commun. 215, 263-269 (2003).

9. C. García, I. Pascual, and A. Fimia, "Obtención de una placa fotosensible como material de registro holográfico," Bol. Soc. Esp. Ceram. Vidrio 39, 435-439 (2000).

10. C. García, A. Fimia, and I. Pascual, "Holographic behavior of a photopolymer at high thicknesses and high monomer concentrations: mechanism of photopolymerization," Appl. Phys. B 72, 311-316 (2001).

11. P. J. Flory, Principles of Polymer Chemistry (Cornell U. Press, Ithaca, N.Y., 1995), pp. 161-177.

12. S. Blaya, "Estudio y desarrollo de nuevos materiales fotopoliméricos aplicados al almacenamiento óptico de información," tesis doctoral (Universidad Miguel Hernández, Elche, Spain, 2001).

13. J. A. Jenney, "Holographic recording with photopolymers," J. Opt. Soc. Am. 60, 1155-1161 (1970).

14. A. Beléndez, R. Fuentes, and A. Fimia, "Noise gratings in thick-phase holographic lenses," J. Opt. (Paris) 24, 99-105 (1993).

15. M. L. Calvo, Óptica Avanzada (Ariel, Barcelona, Spain 2002). 